\title{
On the State of Finite Element Procedures for Forming Processes
}

\author{
Klaus-Jürgen Bathe \\ Massachusetts Institute of Technology, Cambridge, MA USA
}

\begin{abstract}
The solution of forming processes requires reliable and efficient finite element methods to model the various complex physical phenomena encountered. The objective in this presentation is to focus on the current state of finite element methods with respect to reliability and efficiency in modeling forming processes. The finite element procedures pertain to the simulation of sheet metal forming, bulk forming, extrusion and drawing, rolling, welding, cutting processes, etc. It is emphasized that the appropriate finite element methods for a specific problem should be used, and that indeed procedures are available which are effective in many situations. The presentation briefly considers the state of modeling of solids, shell structures, contact conditions, friction, inelastic material response in large strains, thermomechanical coupling and fluid-solid interactions, as encountered in forming process simulations. The solutions of the governing finite element models are obtained using sparse direct or iterative solvers. The oral presentation will include the results of various example simulations.
\end{abstract}

\section{INTRODUCTION}

The finite element method has truly gone through a remarkable development. Since the early inception of the method for engineering use in the 1950's, the method has evolved into a most powerful procedure for general engineering analysis. Finite element programs are used today in a routine manner for the analysis of complex structures, solids, fluids, multiphysics problems and so on. Indeed, the method has become an integral part of computer-aided engineering [ 1 - 4 ].

However, while there is a wide use of the finite element method, the simulation of forming processes still provides a great challenge because of the complex physical phenomena to be simulated. In such analyses, for example, large deformations, large strain anisotropic material behavior, contact conditions including friction, and fluid-structure interactions need be modeled. These conditions and the frequently complex geometries require fine finite element discretizations and considerable computing resources. Of course, as the available finite element procedures, and available hardware, are being advanced, more complex physical conditions can be modeled accurately.
The objective of this presentation is to give a brief overview - indeed just a snapshot - regarding the current state of finite element analysis of forming processes. The presentation focuses on the experiences of the author and therefore gives, in various ways, a rather subjective (indeed personal) view regarding finite element analyses of these complex problems.

\section{THE NEED FOR RELIABLE FINITE ELEMENT METHODS}

A most important aspect of a finite element technique and its program implementation - if the method is to be used in a research study or in an industrial environment - is that the method and implementation be reliable. In particular, the finite element formulation must be mechanistically clear and well-founded, the discretization must be stable and convergent and must not depend on adjustable numerical factors [ 1,5$]$. In addition, of course, efficient solutions are important, in particular, in industrial applications. However, it is quite inappropriate to aim for results in "acceptable" computer run times if these results are not reliable (

CP712, Materials Processing and Design: Modeling, Simulation and Applications, NUMIFORM 2004, edited by S. Ghosh, J. C. Castro, and J. K. Lee

(C) 2004 American Institute of Physics 0-7354-0188-8/04/\$22.00 
and therefore might have to be disregarded in any case ).

Of course, the development of solution methods that are reliable and efficient is a very difficult task. However, for many applications such techniques are now available and we concentrate in this presentation on such procedures.

\section{SOME RESEARCH AND DEVELOPMENTS}

The research and developments briefly mentioned in this section, and pursued by the author and his students and collaborators, are not given in any order of importance, but merely in a natural order from rather general to more specific developments.

\section{Selection of Models}

The selection of an appropriate mathematical model for the solution of a physical problem is the key step of any analysis. It is clearly a difficult task in nonlinear analysis and in particular in forming simulations. Here the possibilities to use hierarchical modeling are very attractive and can lead to effective analyses of complex phenomena $[1,6,7]$.

\section{Mathematical Conditions on Finite Elements}

In many simulations, and notably in the analysis of forming processes, the use of mixed finite elements is necessary. These formulations should satisfy the ellipticity and consistency conditions, and ideally the relevant inf-sup conditions. If the consistency and ellipticity conditions are not satisfied, large unnoticed errors may occur in the finite element simulations.

The importance of the ellipticity and inf-sup conditions has been researched and the shortcomings of rules too simple for an assessment of stability have been delineated $[1,5,8]$.

\section{Shell Analysis}

In many forming processes shell finite element discretizations are used. As mentioned above, the reliability of the shell elements for practical analysis is of primary concern $[1,5,9]$. Our research resulted in the development of the MITC shell elements which have general applicability, are reliable and have good predictive capability $[1,5,10]$. The quadrilateral shell elements are now abundantly used in simulations. Of course, we are still continuing our research to improve these shell elements [11]. We also work to improve, as well, the effectiveness of triangular elements [12] and to include three-dimensional through-the-thickness effects [13]. In metal forming, it can be important to include the through-the-thickness stress and strain effects, and shell elements with quadratic displacement interpolations through the thickness are then effective $[13]$

\section{Inelastic Large Strain Analysis}

Effective large strain elasto-plastic analysis procedures are most important to analyze forming processes. The procedures need to include various ingredients. Firstly, a stable and effective solution scheme for incompressible analysis is necessary. We have developed and use the displacement/ pressure ( $\mathrm{u} / \mathrm{p}$ ) formulation $[1,14]$. Secondly, an effective large strain formulation must be employed. We use the updated Lagrangian Hencky formulation, which is a solution approach based on the total elastic strain computed from the total deformation gradient and an evolution of the plastic deformation gradient. The stresses are calculated using the effective-stressfunction algorithm and consistent tangent matrices are used in the Newton-Raphson iterations [ 1, 15-18 ].

\section{Analysis of Contact Problems}

Many simulations of forming processes involve contact between bodies and an effective contact algorithm must be used. The constraint function method is an attractive scheme for general contact analysis [ 1, 19 - 21]. The solution procedure computes the actual areas of contact and the conditions on the contact surfaces. The normal and tangential ( frictional ) contact conditions are satisfied using constraint functions. The method has been developed for efficient static and implicit dynamic solutions. For increased accuracy, a consistent segment algorithm has been proposed [ 22, 23 ].

\section{Analysis of Fluid Flows and Fluid- Structure Interactions}

During the recent years, we have dedicated a considerable effort to the development of solution techniques for fluid flows and fluid-structure 
interactions [ $24-27]$. These methods can be used to solve very complex fluid flows (modeled by the full Navier-Stokes equations) and fluid-structure interactions in forming processes. The flow-conditionbased interpolation ( FCBI ) procedure with control volumes in the finite element mesh is more effective than earlier published finite element techniques [ 28 ].

\section{Error Evaluation and Mesh Selection}

The automatic evaluation of finite element discretization errors is an important ingredient of a comprehensive analysis tool. Based on the identified discretization errors, the analyst - or an algorithm can construct with the available mesh generation procedures new refined meshes and thus continue the analysis process until the required accuracy is achieved. We use extensively the iso-bands of stresses as a basis of error measures [ 1,29,30 ], but it should be noted that theoretically "sharp" and effective error measures are not yet available [ 31, 32$]$.

\section{Solution of Large Systems}

A most significant aspect is that effective sparse direct and iterative solvers are now available for the simultaneous solution of large sets of finite element equations on $\mathrm{PCs}$ and engineering workstations. Compared to just a decade ago, a reduction of storage by factors of 10 and more, and a reduction of solution times by factors of 100 and more for large systems without taking into account the increase in computer processing speeds - has been reached. This increase in efficiency, added to the increase in efficiency of the finite elements and contact solution methods, and added to the increase in hardware efficiency, makes it possible to simulate today much more complex forming processes than a decade ago [ 2, 3 ].

\section{EXPLICIT VERSUS IMPLICIT TIME INTEGRATION}

The recently reached increased capabilities/ efficiencies in simulating forming processes, referred to above, imply in particular that the physical conditions of forming processes can now be much more accurately simulated than a decade ago. In particular, there is frequently no need to use explicit time integration to simulate slow forming processes.
Because of the difficulties in implicit and static solutions, the common practice has been to use explicit integration not only when the physical process is a fast dynamic phenomenon, but also for almost static situations. Then no iterations to establish equilibrium at the solution steps are performed and a simple forward-marching solution is carried out. However, increased velocities, mass scaling, etc. need be used and the solutions obtained must be studied carefully to assess whether the artificial assumptions did not destroy the accuracy of the predicted response. Indeed, some experimentation with the artificial analysis assumptions may be necessary and lead to a number of additional experimental runs.

Of course, ideally, slow forming processes are simulated using static or implicit dynamic analysis methods. An important point of this presentation is that with the powerful analysis features mentioned above, this more accurate (and appropriate ) modeling can now frequently be employed, e.g. [ 33,34 ].

Hence while, of course, for many fast transient processes, explicit time integration should be used but with reliable finite element methods - slow processes should be modeled using implicit time integration ( or even static analysis conditions ).

\section{CONCLUDING REMARKS}

The objective of this paper was to briefly give some views on the current state of finite element methods available for the simulation of forming processes.

Since the early developments of finite element procedures for forming processes, many advances have taken place, as illustrated in the references below and the references therein. The research and development efforts regarding finite element methods have been very exciting, and these efforts are of course continuing in many research centers. Significant further enhancements must be anticipated, and eight key challenges in finite element research and development have been summarized in the Preface of ref. [2 ]. These challenges include:

- The further improvement of finite element methods, in practically all fields of analysis, but in 
particular for fluid flows, multi-physics and multiscale problems.

- The development of mesh-less methods; some advances are already available, see e.g. refs. [3537] and the references therein.

- The capability to include uncertainties in the input data and solution results.

- The extensive development of virtual laboratories to simulate complete life cycles of structures and systems.

- The teaching of finite element analysis and related fields in an exciting and attractive manner to the young generation.

In all these areas, significant advances must be expected during the years to come that indeed will lead to a "new level of mathematical modeling and numerical solution" that will greatly enhance the possibilities for simulating forming processes.

\section{REFERENCES}

1. Bathe, K. J., Finite Element Procedures, Englewood Cliffs, NJ: Prentice Hall, 1996.

2. Computational Fluid and Solid Mechanics, edited by K. J. Bathe, First M.I.T. Conference on Computational Fluid and Solid Mechanics, Proceedings, Kidlington, Oxford: Elsevier, 2001.

3. Computational Fluid and Solid Mechanics 2003, edited by K. J. Bathe, Second M.I.T. Conference on Computational Fluid and Solid Mechanics, Proceedings, Kidlington, Oxford: Elsevier, 2003.

4. Mackerle, J. "FEM and BEM in the Context of Information Retrieval", Computers \& Structures, 80, $1595-1604$ (2002).

5. Chapelle, D., and Bathe, K. J., The Finite Element Analysis of Shells - Fundamentals, Berlin: Springer Verlag, 2003.

6. Bathe, K. J., Lee, N. S., and Bucalem, M. L., "On the Use of Hierarchical Models in Engineering Analysis", Computer Methods in Applied Mechanics and Engineering, 82, 5-26 (1990).

7. Bucalem, M. L., and Bathe, K. J., Hierarchical Modeling in Finite Element Analysis, Berlin: Springer Verlag, to appear.
8. Bathe, K. J., "The Inf-Sup Condition and its Evaluation for Mixed Finite Element Methods", Computers \& Structures, 79, 243-252, 971 (2001).

9. Chapelle, D., and Bathe, K. J., "Fundamental Considerations for the Finite Element Analysis of Shell Structures", Computers \& Structures, 66, 1936, 711-712 (1998).

10. Bathe, K. J., Iosilevich, A., and Chapelle, D., "An Evaluation of the MITC Shell Elements", Computers \& Structures, 75, 1-30 (2000).

11. Bathe, K. J., Lee, P. S., and Hiller, J. F., "Towards Improving the MITC9 Shell Element", Computers \& Structures, 81, 477-489 (2003).

12. Lee, P. S., and Bathe, K. J., "Development of MITC Isotropic Triangular Shell Finite Elements", Computers \& Structures, in press.

13. Chapelle, D., Ferent, A., and Bathe, K. J., "3DShell Elements and Their Underlying Mathematical Model", Mathematical Models \& Methods in Applied Sciences, 14, 1-38 (2004).

14. Sussman, T., and Bathe, K. J., "A Finite Element Formulation for Nonlinear Incompressible Elastic and Inelastic Analysis", Computers \& Structures, 26, 357-409 (1987)

15. Eterovic, A. L., and Bathe, K .J., "A HyperelasticBased Large Strain Elasto-Plastic Constitutive Formulation with Combined Isotropic-Kinematic Hardening Using the Logarithmic Stress and Strain Measures", International Journal for Numerical Methods in Engineering 30, 1099-1114 (1990).

16. Gabriel, G., and Bathe, K. J., "Some Computational Issues in Large Strain ElastoPlastic Analysis", Computers \& Structures, 56, 249-267 (1995).

17. Montans, F. J., and Bathe, K. J., "On the Stress Integration in Large Strain Elasto-Plasticity", in Computational Fluid and Solid Mechanics 2003 edited by K. J. Bathe, Kidlington, Oxford: Elsevier, 2003

18. Montans, F. J., and Bathe, K. J., "Computational Issues in Large Strain Elasto-Plasticity: An Algorithm for Mixed Hardening and Plastic Spin", Inernational Journal for Numerical Methods in Engineering, submitted.

19. Eterovic A. L., and Bathe, K. J., "On the Treatment of Inequality Constraints Arising from Contact Conditions in Finite Element Analysis", Computers \& Structures, 40, 203-209 (1991).

20. Bathe, K. J., and Bouzinov, P., "On the Constraint Function Method for Contact 
Problems", Computers \& Structures, 64, 1069 1085 (1997).

21. Pantuso, D., Bathe, K. J., and Bouzinov, P. A., "A Finite Element Procedure for the Analysis of Thermo-Mechanical Solids in Contact", Computers \& Structures, 75, 551-573 (2000).

22. Bathe, K. J., and Brezzi, F., "Stability of Finite Element Mixed Interpolations for Contact Problems", Atti della Accademia Nazionale dei Lincei, s. 9, 12, 159-166 (2001).

23. El-Abbasi, N., and Bathe, K. J., "Stability and Patch Test Performance of Contact Discretizations and a New Solution Algorithm", Computers \& Structures, 79, 1473-1486 (2001).

24. Bathe, K. J., Zhang, H., and Ji, S., "Finite Element Analysis of Fluid Flows Fully Coupled with Structural Interactions", Computers \& Structures, 72, 1-16 (1999).

25. Rugonyi, S., and Bathe, K. J., "On the Finite Element Analysis of Fluid Flows Fully Coupled with Structural Interactions", Computer Modeling in Engineering \& Sciences, 2, 195-212 (2001).

26. Bathe, K. J., and Zhang, H., "A Flow-ConditionBased Interpolation Finite Element Procedure for Incompressible Fluid Flows", Computers \& Structures, 80, 1267-1277 (2002).

27. Bathe, K. J., and Zhang, H., "Finite Element Developments for General Fluid Flows with Structural Interactions", International Journal for Numerical Methods in Engineering, in press.

28. Hendriana, D., and Bathe, K. J., "On Upwind Methods for Parabolic Finite Elements in Incompressible Flows", International Journal for Numerical Methods in Engineering, 47 (1-3), 317 $330(2000)$.

29. Sussman, T., and Bathe, K. J., "Studies of Finite Element Procedures - Stress Band Plots and the Evaluation of Finite Element Meshes", $J$. Engineering Computations 3, 178-191 (1986).

30. Lee, N. S., and Bathe, K. J., "Error Indicators and Adaptive Remeshing in Large Deformation Finite Element Analysis", Finite Elements in Analysis and Design 16, 99 -139 (1994).

31. Hiller, J. F., and Bathe, K. J., "On Higher-OrderAccuracy Points in Isoparametric Finite Element Analysis and an Application to Error Assessment", Computers \& Structures, 79, 1275-1285 (2001).

32. Grätsch, T., and Bathe, K. J., "The State of A Posteriori Error Estimation Techniques for Use in
Practical Finite Element Analysis", Computers \& Structures, submitted

33. Bathe, K. J., Walczak, J., Guillermin, O. Bouzinov, P., and Chen, H., "Advances in Crush Analysis", Computers \& Structures, 72, 31-47 (1999).

34. Kawka, M., and Bathe, K. J., "Implicit Integration for the Solution of Metal Forming Processes", in Computational Fluid and Solid Mechanics edited by K.J. Bathe, Kidlington, Oxford: Elsevier 2001.

35. De, S., and Bathe, K. J., "The Method of Finite Spheres with Improved Numerical Integration", Computers \& Structures, 79, 2183-2196 (2001).

36. De, S., and Bathe, K. J., "Displacement/Pressure Mixed Interpolation in the Method of Finite Spheres", International Journal for Numerical Methods in Engineering, 51, 275-292 (2001).

37. Hong, J. W., and Bathe, K. J., "Coupling and Enrichment Schemes for Finite Element and Finite Sphere Discretizations", Computers \& Structures, to appear. 